\title{
Precoding based on Signal-to-leakage and Noise Ratio to Reduce ICI in MIMO-OFDM Systems
}

\author{
Ambuj Mehrish \\ National Institute of Technology \\ Hamirpur
}

\author{
Vikash Kumar \\ National Institute of Technology \\ Hamirpur
}

\author{
Ashish Goswami \\ National Institute of Technology \\ Hamirpur
}

\begin{abstract}
Current trends in the development of high-data-rate wireless systems focus on the integration of orthogonal frequencydivision multiplexing (OFDM) and multiple-input?multiple-output (MIMO).The combination of OFDM and the Multiple-InputMultiple-Output (MIMO) technique represents a promising candidate for future broadband wireless systems. This paper addresses the Inter Carrier Interference (ICI) issue in multiuser MIMO-OFDM systems operating in time-varying frequency selective channel environments.In our proposed method precoding based on signal-to-leakage-and-noise ratio (SLNR) is considered.In this paper model using precoding in MIMOOFDM is introduced.Based on the model algorithm for precoding matrix is developed.Simulation results confirms reduction in ICI without increasing complexity of a system.
\end{abstract}

\section{General Terms:}

Wireless Communication, Multiple Input Multiple Output, Otrhogonal Frequency Division Multiplexing

\section{Keywords:}

Signal-to-leakage noise ratio, ICI, Precoding

\section{INTRODUCTION}

The explosive growth of wireless communications is creating the demand for high-speed, reliable, and spectrally efficient communication over the wireless medium. There are several challenges in attempts to provide high-quality service in this dynamic environment.These pertain to channel time-variation and the limited spectral bandwidth available for transmission.Channel variations arise due to the presence of an unknown carrier frequency offset (CFO). While sub carriers in OFDM are orthogonal in the presence of the time-invariant multipath channel, the rapid channel variations in a symbol period destroy the orthogonality among sub carriers, which results in inter carrier interference (ICI) $[1,4]$.

MIMO-OFDM combines the simple equalization of OFDM modulation with the capacity, diversity, and array gain of MIMO communication.Currently MIMO-OFDM is being considered in various multi-user systems including high-speed wireless local area networks and next generation cellular systems. The dominant sources of interference in a multi-user MIMO-OFDM system are multiple access interference, co-antenna interference which is the interference caused by the signals from multiple transmit antennas of a given user being received on the same receive antenna, and inter-carrier interference (ICI). If we do not take the measure to overcome the disturbance, there will be extremely seriously affects to the system performance.

While the benefits of MIMO are realizable when the receiver alone knows the communication channel, these are further enhanced when the transmitter also knows the channel. The value of transmit channel knowledge can be significant. For example, in a four-transmit two-receive antenna system with independent identically distributed (i.i.d.) Rayleigh flat-fading,transmit channel knowledge can more than double the capacity at?5dB signal-tonoise ratio (SNR) and add $1.5 \mathrm{~b} / \mathrm{s} / \mathrm{Hz}$ additional capacity at $5 \mathrm{~dB}$ SNR. Such SNR ranges are common in practical systems such as WiFi and WiMax applications. In a non-i.i.d. channel (such as correlated Rician fading), channel knowledge at the transmitter offers even greater leverage in performance. Therefore, exploiting transmit channel side information is of great practical interest in MIMO wireless. In this article, we assume full channel knowledge at the receiver and study how channel-side information at the transmitter (CSIT) can be used to improve link performance. While the origins of using CSIT at the transmitter or precoding dates back to Shannon, MIMO precoding has been an active research area during the last decade, fueled by applications in commercial wireless technology.

Recently, the most commonly used method to reduce ICI is frequency-domain equalization [5], which uses training signals. The time-domain windowing method [6] can also reduce ICI through multiplying the transmitted time-domain signals by a well-designed windowing function. In ICI self-cancellation scheme[7][8], the ICI is cancelled out by repeatedly modulating a symbol on two adjacent sub-carriers with a 180 degree phase difference between them, but the cost is the lose of the bandwidth. [9] uses precoding to reduce the ICI of OFDM, but it dose not consider the case of MIMO-OFDM and multi-user, and the precoding based on ZF has a restriction on system configuration.

Precoding is a processing technique that exploits CSIT by operating on the signal before transmission. For many common forms of partial CSIT, a linear precoder is optimal from an information theoretic view point [4]?[6]. A linear precoder essentially 
functions as a multimode beamformer, optimally matching the input signal on one side to the channel on the other side. It does so by splitting the transmit signal into orthogonal spatial eigenbeams and assigns higher power along the beams where the channel is strong but lower or no power along the weak. Precoding design varies depending on the types of CSIT and the performance criterion.

Although the term precoding is sometimes used in the literature to represent any transmit processing besides channel coding, we clarify its use here to strictly mean the transmit signal processing that involves CSIT. MIMO techniques without CSIT are clarified as space-time (ST) coding. Since the work of Shannon, more recent results show that, for a flat-fading wireless channel, provided a mild condition that the current channel state is independent of the previous CSIT when given the current CSIT, the capacity can be achieved by CSIT-independent coding together with CSIT-dependent linear precoding . The linear precoder directs signal spatially and allocate power in a water-filling fashion over both space and time. Power allocation over time can slightly increase the capacity of a fading channel at low SNRs, but has diminishing impact as the SNR increases beyond roughly $15 \mathrm{~dB}$ [9]. Depending on the antenna configuration, allocation over space, on the other hand, can significantly increase the capacity at all SNRs. This motivates precoding designs to exploit spatial CSIT

In this paper, we combine the OFDM with MIMO technique, discuss a new precoding in multi-user MIMO-OFDM downlink. The main idea of this scheme is that the design of precoding vectors is based on sub-carriers channel. The multi-user MIMO system will be changed to single-user MIMO system according to the design of precoding vector with every sub-carrier channel. At last the ICI will be eliminated well and the noise will be restrained.

\section{PRECODING SYSTEM MODEL}

We consider MIMO-OFDM system with where $\mathrm{M}$ antennas are located at the BS and $\mathrm{N}$ antennas are located at the kth mobile station (MS).There are $\mathrm{L}$ number of sub carriers. The modulate signal of user $\mathrm{k}$ is converted into $\mathrm{N}$ parallel data streams at first. $d_{k, i}$ $(\mathrm{i}=1,2, \ldots \mathrm{M})$.Then the data streams will multiple the precoding vectors $P_{k, i}$ respectively.System performance is improved by creating a balance between eliminating the co channel interference and and the noise.After that, the data stream will be converted to 1 parallel data streams respectively. The $\mathrm{L}$ data streams are corresponding to $\mathrm{L}$ sub-carriers, and they will have a IFFT conversion.In order to mitigate the effects of channel delay spread, a guard interval comprised of either a $\mathrm{CP}$ or suffix is appended to the sequence. At last the signal will be transmitted from transmission antenna.

At the receiver, the received data stream will be removed $\mathrm{CP}$ at first, the useful data will be distilled to have a FFT conversion. At last, we get the estimate data according to the decoding vector $d_{k}$ The received signal (don?t consider the IFFT and FFT conversion) of user $\mathrm{k}$ at sub-carrier 1 can be written by

$$
r_{k, l}=H_{k, l} \sum_{k=1}^{k} P_{k, l} d_{k, l}+n_{k, l}
$$

Where $H_{k, l}$ is the channel matrix from user k to base station at subcarrier $1 . d_{k, l}$ is the transmitted signal from user $\mathrm{k}$ at sub-carrier 1 and $n_{k, l}$ is the noise.

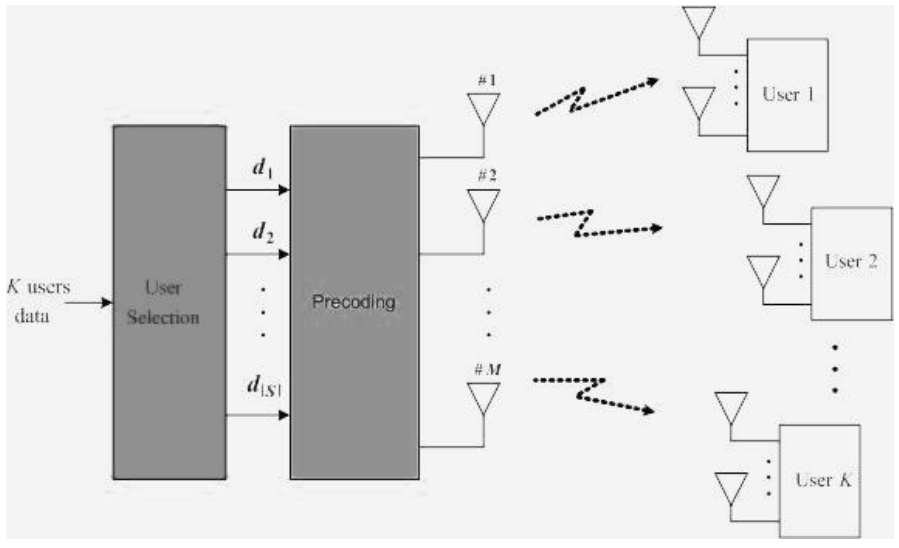

Fig. 1. Multi-user MIMO-OFDM with Precoding
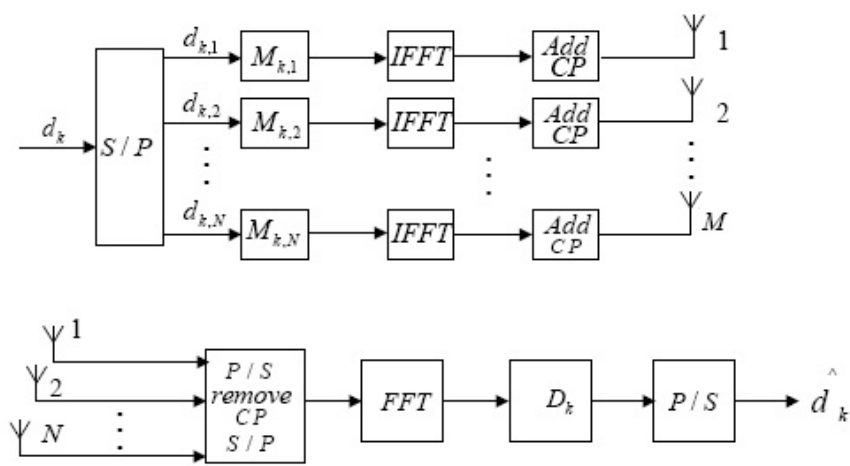

Fig. 2. System model of a multi-user MIMO-OFDM with precoding

\section{ALGORITHM FOR PRECODING MATRIX}

Equation (1) cab be written as

$$
r_{k, l}=H_{k, l} P_{k, l} d_{k, l}+\sum_{k=1}^{k} H_{k, l} P_{k, l} d_{k, l}+n_{k, l}
$$

Second term in equation 2 is co channel interference.Using Zero forcing algorithm to cancel CCI,so

$$
H_{j, l} P_{k, l}=0, j=1, \ldots k, j \neq k
$$

But the ZF solution imposes a strong condition on the system configuration, it needs to require

$$
N>\max \left\{\sum_{i=1, i \neq k}^{k} M_{i}\right\}
$$

The CCI is interference at desire signal caused by all other signals. Now we consider interference caused by desired signal on the remaining signal. Which is a leakage.The leakage for user $\mathrm{k}$ at subcarrier 1 is written as

$$
\sum_{i=1, i \neq k}^{k}\left\|H_{i, l} P_{k, l}\right\|^{2}
$$


The SLNR of user $k$ at sub-carrier 1 is

$$
\begin{aligned}
S L N R_{k, l} & =\frac{\left\|H_{k, l} P_{k, l}\right\|^{2}}{M \sigma_{k, l}^{2}+\sum_{i=1, i \neq k}^{k}\left\|H_{i, l} P_{k, l}\right\|^{2}} \\
& =\frac{\left\|H_{k, l} P_{k, l}\right\|^{2}}{M \sigma_{k, l}^{2}+\left\|\tilde{H}_{i, l} P_{k, l}\right\|^{2}}
\end{aligned}
$$

The optimum $P_{k, l}$ is generalized eigenvector corresponding to the largest eigenvalue of matrix pair $\left[H_{k, l}^{H} H_{k, l}, \sum_{i=1, i \neq k}^{k} H_{i, l}^{H} H_{i, l}+\right.$ $\left.\sigma_{k, l}^{2} I\right]$. When the latter matrix is invertible, the solution can also be expressed as

$$
\begin{gathered}
P_{k, l}=\arg \max _{P_{k, l \in C}} S L N R_{k, l} \\
=\max \text { eigenvector }\left(\left(M \sigma_{k, l}^{2} I+\tilde{H_{k, l}^{*}} \tilde{H_{k, l}}\right)^{-1} H_{k, l}^{*} H_{k, l}\right)
\end{gathered}
$$

Where $\tilde{H_{k, l}}=\left[H_{1, l} \ldots H_{k-1, l} H_{k+1, l} \ldots . . H_{K, l}\right]^{T}$

Since we have not consider FFT and IIFT in our above calculation .Let $\mathrm{F}$ denote FFT conversion matrix and $F^{H}$ as IIFT conversion matix .Than Equivalant channel is given by

$$
H^{\prime}=F^{H} H F
$$

After performing above substitution in equation (9).Precoding ma-

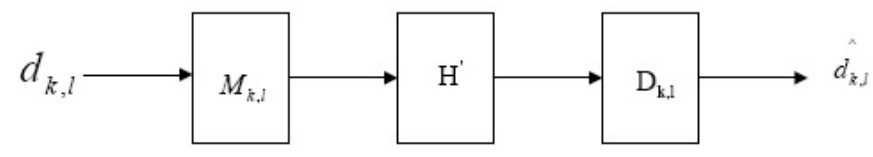

Fig. 3. The equivalent single-user MIMO link

trix can be expressed as

$$
\begin{array}{r}
P_{k, l}=\max \text { eigenvector }\left(\left[M \sigma_{k, l}^{2} I+\left(F^{H} H_{k, l} \tilde{l}\right) *\left(F^{H} \tilde{H_{k, l}} F\right)\right]^{-1}\right. \\
\left.\left(F^{H} H_{k, l} F\right)^{*}\left(F^{H} H_{k, l} F\right)\right)
\end{array}
$$

\section{SIMULATION RESULTS}

Fig 4 compares the simulated bit error rate (BER) in MIMO OFDM system using different system configuration.MIMO OFDM without precoding, MIMO OFDM with ZF precoding and SLNR based precoding are compared.

Fig 5 compares the simulated bit error rate(BER)with SLNR based precoding for different transmit antennas .

The number of the subcarriers is chosen to be $\mathrm{L}=128$, and the length of the cyclic prefix is $\mathrm{Np}=\frac{L}{8}$. The QPSK constellation is adopted, and the bit energy is $E b=\frac{E s}{2}$. We use a 2-tap fading channel with an exponential delay power spectrum ,ie, $\sigma_{l}^{2}=\frac{\exp \frac{-l}{L}}{\sum_{l=0}^{L-1} \sigma_{l}^{2}} \mathrm{~L}=2$, and $1=0,1$. Each channel tap is a complex Gaussian random process independently generated with the classical Doppler spectrum based on the Jakes's model[10].The delay of the first tap is zero, and the delay of the second tap is generated from the set $\left\{T_{c} 2 T_{c}, \ldots L T_{c}\right\}$ with equal probability.

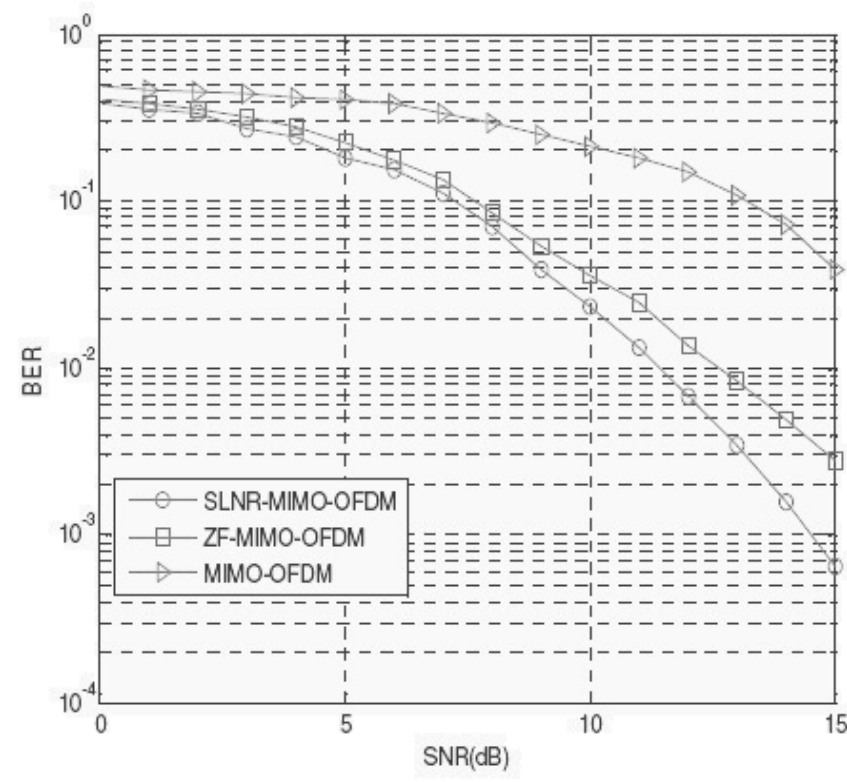

Fig. 4. BER Performance of Precoded MIMO OFDM in comparison with conventional MIMO OFDM

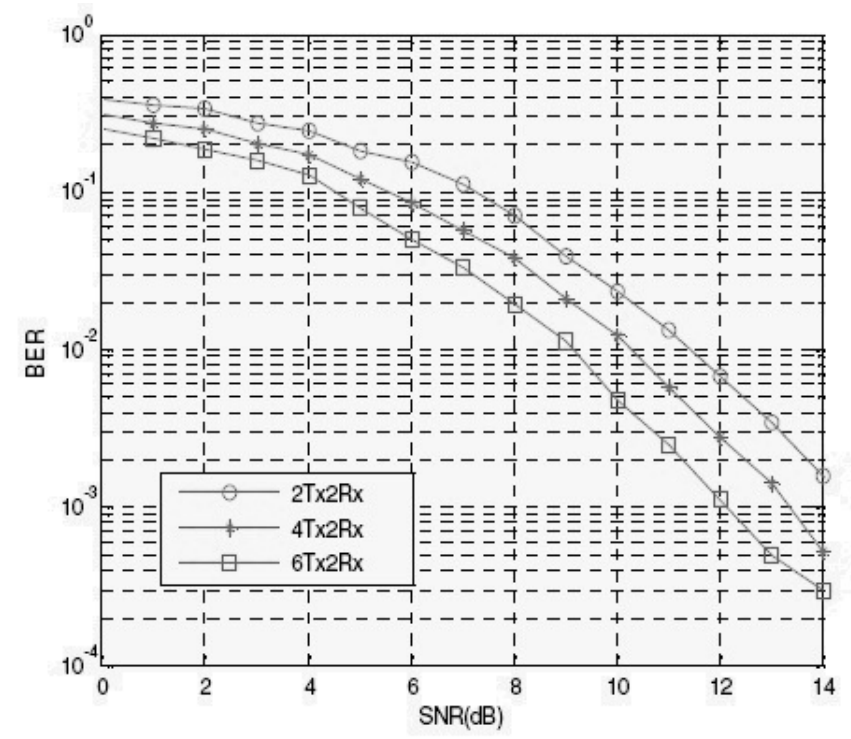

Fig. 5. BER Performance of Precoded MIMO OFDM with different transmit antenna

\section{CONCLUSION}

The ICI can be restrained well in MIMO-OFDM with precoding based on the SLNR. We can acquire the frequency diversity gain and the Doppler diversity gain,enhance the signal energy and improve the SNR according to selecting appropriate precoding matrix actually. 


\section{REFERENCES}

[1] Y. G. Li and L. J. Cimini, "Bounds on the inter-channel interference of OFDM in time-varying impairments" IEEE Trans. Commun., vol. 49, pp. 401-404, Mar. 2001

[2] F. Ling, "Matched filter-bound for time-discrete multipath Rayleigh fading channels" IEEE Trans.Commun., vol. 43, pp. 710713, Feb./Mar./Apr.1995.

[3] J. G. Proakis, Digital Communications. McGraw- Hill, 1995.

[4] M. Russell and G. L. S. Stuber, "Inter channel interference analysis of OFDM in a mobile environment" in Proc. of VTC'95, Chicago, Illinois, pp. 820-824, July 1995.

[5] Hongnian Xing, Petri Jarske, Frequency Domain Equalization for the Multicarrier Spread Spectrum System in Multipath Fading Channel, Tampere University of Technoligy, Digtial Media Institute?1994

[6] R. Zhang, T. T. Tjhung, Seniro Member, IEEE, H. J. Huu, P. $\mathrm{He}$, Window Function and Interpolation Algorithm for OFDM Frequency-Offset Correction, IEEE TRANSACTIONS ON VEHICULAR TECHNOLOGY, VOL.52, NO. 3, MAY 2003

[7] Yuping Zhao, Jean-Damien Leclercq, Sven-Gustav Haggman, Intercarrier Interference Compression in OFDM Communication Systems by Using Correlative Coding,IEEE COMMUNICATIONS LETTERS,VOL.2,NO.8,AUGUST 1998

[8] Alireza Seyedi, Gary J. Saulnier, General ICI Self-Cancellation Scheme for OFDM Systems, IEEE TRANSACTIONS ON VEHICULAR TECHNOLOGY,VOL. 54, NO. 1, JANUARY 2005

[9] Jiang Linlin and Xie Xianzhong, A new way to reduce ICI of OFDM system with precoding, IEEE ICCAS2007, Vol1,p288p291

[10] W. C. Jakes, Microwave Mobile Communications.New York: Wiley, 1974 